\title{
Energy scavenging from ambient sources based on ferroelectric materials
}

\section{Daniel Guyomar, Mickaël Lallart}

Daniel Guyomar, Mickaël Lallart, "Energy scavenging from ambient sources based on ferroelectric materials," Proc. SPIE 7295, Health Monitoring of Structural and Biological Systems 2009, 729523 (10 April 2009); doi: 10.1117/12.817506

Event: SPIE Smart Structures and Materials + Nondestructive Evaluation and Health Monitoring, 2009, San Diego, California, United States 


\title{
Energy Scavenging From Ambient Sources Based On Ferroelectric Materials
}

\author{
Daniel Guyomar and Mickaël Lallart \\ Université de Lyon, INSA-Lyon, LGEF EA 682, F-69621, Villeurbanne, FRANCE;
}

\begin{abstract}
This paper addresses the problem of piezoelectric conversion enhancement from mechanical to electrical energy and illustrates this improvement on vibration control and health monitoring applications. Considering a mechanical structure equipped with piezoelements, it can be shown that a non-linear processing (SSHI) of the piezoelement output voltage improves significantly the energy conversion. This non-linear processing simply corresponds to short-circuit the voltage for a brief period of time when the voltage reaches a maximum or minimum. Technically, a non-linear switch is added in parallel with the piezoelement, thus the piezovoltage, in front of the rectifier, increases and consequently more energy flows to the storage capacitance. The harvested energy is nine times higher than the standard approach. The influence of piezo-material characteristics will be described. Extension of the non-linear approach to harvesting in the pulse regime leads also to a performance increase specifically for low coupled structure which is mostly the case. After an overview of the basic principles, the presentation will go over new extensions of the SSHI approach to increase the output power, to make it independent of the resistive load or to minimize the voltage drop effect in the rectifier. The SSHI extension to heat harvesting will be also introduced.
\end{abstract}

Keywords: Piezoelectric, nonlinear treatment, vibration damping, energy harvesting, self-powered

\section{INTRODUCTION}

Thanks to recent advances in microelectronics and energy conversion, the possibility of designing truly selfpowered devices has become no longer chimerical. Such a trend is besides encouraged by an increasing demand in terms of autonomous sensors and sensor networks from the industrial field. Self-powered systems that harvest their energy from their near environment find their application in many domains, such as Structural Health Monitoring (SHM) of building or aeronautic structures. ${ }^{1,2}$ Another key applications concern the biomedical field or home automation (e.g. autonomous, self-powered wireless switch ${ }^{3}$ ).

Various sources can be used as energy tank for supplying such systems, ${ }^{4}$ such as solar, magnetic or thermal. Among all of the available environmental energies, mechanical source through vibrations is one of the most commonly available, and particularly suitable for conversion into electrical energy using the piezoelectric effect. Therefore Piezoelectric Electrical Generators (PEGs) have been intensively studied ${ }^{5-8}$ as denoted by the reviews done by Sodano ${ }^{9}$ or Priya. ${ }^{10}$ Moreover piezoelectric elements offer high power densities as well as a very good integration potential, making them particularly suitable for the conception of MEMS microgenerators.

However, the amount of scavenged vibrational energy is still limited which therefore prevents from the implementation of numerous and/or complex functions. Typically, the amount of harvested power is within the range of few tens of microwatts to a few milliwatts, consequently limiting self-powered devices to sensing functions (rather than actuating functions).

In order to increase the amount of converted energy, and therefore the output power of the microgenerator, Guyomar, Richard et al. have proposed an original approach based on a simple nonlinear processing of the output voltage of piezoelements. Initially dedicated to vibration damping, ${ }^{11,12}$ such a technique has also been

Further author information: (Send correspondence to D. Guyomar)

D. Guyomar: E-mail: daniel.guyomar@insa-lyon.fr

M. Lallart: E-mail: mickael.lallart@insa-lyon.fr

Health Monitoring of Structural and Biological Systems 2009, edited by Tribikram Kundu,

Proc. of SPIE Vol. 7295, 729523 - @ 2009 SPIE · CCC code: 0277-786X/09/\$18 · doi: 10.1117/12.817506

Proc. of SPIE Vol. 7295 729523-1 


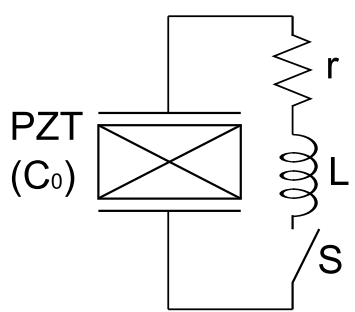

Figure 1. Nonlinear treatment schematics.

proved to be particularly efficient for vibrational energy harvesting, ${ }^{13,14}$ leading to the so-called Synchronized Switch Harvesting on Inductor (SSHI) approach.

This technique is addressed in this paper, whose aims are twofold. It first goal consists in explaining the nonlinear treatment principles, and exposing the underlying mechanisms that lead to a magnification of the energy conversion. Secondly, it aims at exposing the different techniques and their enhancement derived from this nonlinear treatment. The paper is organized as follows. Section 2 exposes the principles of the nonlinear treatment, and explains in details the mechanisms of the conversion enhancement. The application of this method to the vibration damping problem in a semi-passive or semi-active manner in detailed in Section 3. Then the original extension to energy harvesting is exposed in Section 4. The different evolutions of this nonlinear energy harvesting technique, as well as their advantages and drawbacks, are then described in Section 5. Finally, Section 6 briefly concludes the paper.

\section{NONLINEAR CONVERSION ENHANCEMENT PRINCIPLES}

This Section aims at exposing the general principles of the conversion enhancement by nonlinear treatment, as well as detailing using a new approach the conversion mechanisms that lead to an artificial enhancement of the coupling coefficient.

\subsection{Principles}

When dealing with piezoelectric elements, the available electrical energy (converted from mechanical energy) can be obtained considering the electric field or equivalently the piezovoltage. Particularly, it can be shown that this energy is a function of the squared voltage. Therefore an increase of this voltage would cause an energy increase for the same input energy of the system, and consequently the conversion ratio between the mechanical energy and the electrical energy would be improved.

A way to obtain such a voltage increase without any external energy supply consists in intermittently connecting, using a digital switch $S$, the piezoelectric element to an electrical circuit made of a resistance $r$ and possibly an inductance $L$, that shapes an oscillating network with the piezoelectric element - this latter having a capacitive behavior (with a clamped capacitance $C_{0}$ ) - as depicted in Fig. 1.

When the piezoelement is connected to the $R L$ circuit, its voltage starts oscillating around zero with a pseudofrequency relatively high compared to the vibration frequency. Therefore, if the piezoelement is disconnected from the circuit a half period latter, the voltage sign has been changed. This voltage inversion effect (which is imperfect due to internal losses) can thereafter be used in order to artificially increase the voltage. Indeed, the capacitive nature of the piezoelement ensuring the continuity of the voltage, the effect of switching is equivalent to a change in the initial condition of the voltage. Therefore in order to ensure the greater increase, the switching instant has to be chosen such as the absolute voltage is maximal (i.e. a voltage extremum), shaping typical waveforms depicted in Fig. 2.

As well, such an approach offers a very interesting adaptiveness thanks to the extremum detection principles, allowing operations on a wide frequency band (and therefore this technique is insensitive to frequency drifts). The implementation of such a treatment can also be truly self-powered, ${ }^{15}$ using a very small part (typically $5 \%$ ) of the electrostatic energy available on the piezoelectric element. 


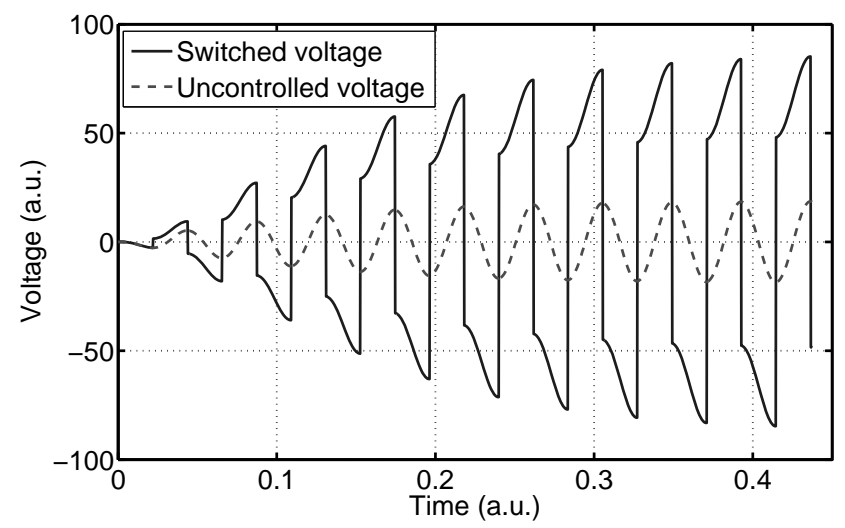

Figure 2. Nonlinear treatment voltage waveforms.

Finally, it can be noted that such a treatment is independent from the physical effect of conversion, as long as the output electrical quantity (i.e. voltage or current) is varying. Hence, such an approach can also be applied to magnetic energy conversion ${ }^{16}$ or thermal time variation using the pyroelectric effect ${ }^{17}$ without any conceptual problem.

\subsection{Conversion mechanisms}

It is proposed here to expose a deep investigation of the mechanisms of the conversion using the previously exposed nonlinear approach.

\subsubsection{Physical approach}

Starting from the piezoelectric constitutive equations:

$$
\left\{\begin{array}{l}
d D=\delta d T+\epsilon^{T} d E \\
d S=s^{E} d T+\delta^{t} d E
\end{array}\right.
$$

where $T, S, E$ and $D$ respectively denote the stress, strain, electric field and induction field, and $\delta, \epsilon^{T}, s^{E}$ the piezoelectric coefficient (with ${ }^{t}$ the transpose operator), permittivity under constant stress and compliance under constant electric field, the electrostatic energy $E_{e l}$ available on the piezoelectric element under constant induction field (i.e. open circuit condition) and uniaxial solicitation can be expressed as:

$$
E_{e l}=\frac{1}{2} \epsilon^{S} E^{2}=\frac{1}{2} \frac{\delta^{2}}{\epsilon^{T}} T^{2}
$$

Therefore, without any particular treatment in open-circuit condition, the maximal value of the electrostatic energy is given by:

$$
\left(E_{e l}\right)_{\text {open }}=\frac{1}{2} \frac{\delta^{2}}{\epsilon^{T}} T_{M}^{2}
$$

When applying the nonlinear operation, the initial condition change produced by the switching process can be seen as a pre-stress $T_{0}$ whose value in steady-state condition is given by:

$$
T_{0}=\frac{2 \gamma}{1-\gamma} T_{M}
$$

with $\gamma$ the inversion coefficient (i.e. the ratio between the absolute value of the voltage just after the switching process and the absolute value of the voltage just before the switching process). Therefore the maximal value of the electrostatic energy with the switching process yields: 


$$
\left(E_{e l}\right)_{s w}=\frac{1}{2} \frac{\delta^{2}}{\epsilon^{T}} \frac{4(1+\gamma)^{2}}{(1-\gamma)^{2}} T_{M}^{2}
$$

which is $\frac{4(1+\gamma)^{2}}{(1-\gamma)^{2}}$ times greater than in the open circuit case. Such a conversion magnification can also be expressed using the equivalent coupling coefficient:

$$
k_{e q}^{2}=\frac{\text { electrical energy }}{\text { total mechanical energy }}=\frac{4(1+\gamma)^{2}}{(1-\gamma)^{2}} \frac{\delta^{2}}{\epsilon^{T} s^{E}}=\frac{4(1+\gamma)^{2}}{(1-\gamma)^{2}} k_{o p e n}{ }^{2}
$$

where $s^{E}$ refers to the compliance with constant electric field and $k_{\text {open }}{ }^{2}$ the squared coupling coefficient with no particular process.

\subsubsection{Spring-mass-damper model approach}

The effect of the conversion enhancement can also be demonstrated using a commonly used model based on an electromechanically coupled spring-mass-damper system ${ }^{12}$ whose governing equations are:

$$
\left\{\begin{array}{l}
M \ddot{u}+C \dot{u}+K_{E} u=F-\alpha V \\
I=\alpha \dot{u}-C_{0} \dot{V}
\end{array}\right.
$$

where $F, u, V$ and $I$ represent the driving force, displacement at a given position, piezovoltage and current flowing out of the piezoelement, respectively. $M, C$ and $K_{E}$ are defined as the equivalent dynamic mass, structural damping coefficient and short-circuit stiffness, and $\alpha$ and $C_{0}$ the force factor and blocking capacitance, respectively.

Multiplying Eq. (7) by the velocity $\dot{u}$ and integrating over one vibration period $T$ therefore yields the energy balance:

$$
\frac{1}{2} M\left[\dot{u}^{2}\right]_{0}^{T}+C \int_{0}^{T} \dot{u}^{2} d t+\frac{1}{2} K_{E}\left[u^{2}\right]_{0}^{T}=\int_{0}^{T} F \dot{u} d t-\alpha\left(\int_{0}^{T} V I d t+\frac{1}{2} C_{0}\left[V^{2}\right]_{0}^{T}\right)
$$

The terms in brackets of the right side member corresponds to the converted electrical energy. $\int_{0}^{T} V I d t$ is the energy exchanged with the control circuit (i.e. the switching losses), while $\frac{1}{2} C_{0}\left[V^{2}\right]_{0}^{T}$ is the energy converted to electrostatic energy. Referring to Fig. 2, the electrostatic energy increases during the transient stage, reflecting the conversion enhancement.

\section{APPLICATION TO VIBRATION DAMPING: THE SSD TECHNIQUE}

The previous Section introduced the concept of the nonlinear treatment and its effect in energy conversion enhancement. In this Section is proposed the application of such a technique for vibration damping purposes. While traditional approaches for vibration damping consists in purely passive techniques that necessitates huge inductance and are very sensitive to frequency drifts, or active techniques that have huge energy requirements, it is possible to take advantage of the conversion enhancement and robustness of the previously exposed processing, leading to the concept of Synchronized Switch Damping (SSD).

Moreover, in order to limit the switching losses and therefore enhance the energy conversion further, a voltage source $V_{S}$ can be added in the switching circuit, as depicted in Fig. 3. Actually two switches and voltage sources with opposed sign has to be implemented for positive and negative switches. As well, the method using voltage sources is no longer semi-passive as it requires an external energy source.

Particularly, assuming a monomodal system that can be modeled by a spring-mass-damper system, the expression of the displacement in open-circuit condition $(I=0)$ for low damped systems yields from Eqs. (7): 


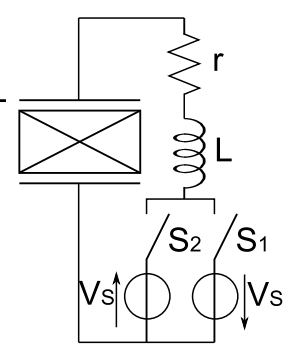

Figure 3. SSD schematics.

$$
u_{o c}=\frac{F_{M}}{-M \omega^{2}+j C \omega+K_{D}}
$$

with $\omega$ the angular frequency and $K_{D}$ the open circuit stiffness defined as $K_{D}=K_{E}+\alpha^{2} / C_{0}$, and assuming null initial conditions. When applying the nonlinear treatment, it can be shown ${ }^{11}$ that the voltage can be expressed as the sum of a voltage proportional to the displacement and a piecewise constant voltage in phase with the speed $\dot{u}$ :

$$
V_{s w}=\frac{\alpha}{C_{0}} u+\frac{1+\gamma}{1-\gamma}\left(\frac{\alpha}{C_{0}} u_{M}+V_{S}\right) \operatorname{sgn}(\dot{u})
$$

where $u_{M}$ denotes the displacement magnitude.

Assuming that only the first harmonic of this voltage has an effect on the displacement (i.e. the structure filters high harmonics), the expression of the displacement yields, from Eqs. (7) and (10):

$$
u_{s w}=\frac{F_{M}-\frac{4}{\pi} \frac{1+\gamma}{1-\gamma} \alpha V_{S}}{-M \omega^{2}+j\left(C \omega+\frac{4}{\pi} \frac{1+\gamma}{1-\gamma} \frac{\alpha^{2}}{C_{0}}\right)+K_{D}}
$$

Particularly, when considering an excitation at the resonance angular frequency $\omega_{0}$, the expression of the attenuation is given by:

$$
\frac{u_{s w}}{u_{c o}}=\frac{1-\frac{4}{\pi} \frac{1+\gamma}{1-\gamma} \alpha \frac{V_{S}}{F_{M}}}{1+\frac{1}{C \omega_{0}} \frac{4}{\pi} \frac{1+\gamma}{1-\gamma} \frac{\alpha^{2}}{C_{0}}}=\frac{1}{1+\frac{4}{\pi} \frac{1+\gamma}{1-\gamma} k^{2} Q_{M}}\left(1-\frac{4}{\pi} \frac{1+\gamma}{1-\gamma} \alpha \frac{V_{S}}{F_{M}}\right)
$$

with $k^{2}$ the global electromechanical coupling coefficient and $Q_{M}$ the mechanical quality factor. This relationship shows that, when using a non null voltage source, there exists a minimal value of the driving force magnitude for ensuring the system stability. ${ }^{18} \mathrm{~A}$ way to tackle this drawback consists in tuning the value of $V_{S}$ to the displacement magnitude such as:

$$
V_{S}=\beta \frac{\alpha}{C_{0}} u_{M}
$$

where $\beta$ is a user-defined coefficient. Therefore the expression of the attenuation turns to:

$$
\frac{u_{s w}}{u_{c o}}=\frac{1}{1+(1+\beta) \frac{4}{\pi} \frac{1+\gamma}{1-\gamma} k^{2} Q_{M}}
$$

which is equivalent to consider a system with a null voltage but an enhanced inversion coefficient:

$$
\gamma_{e q}=\frac{(1+\beta)(1+\gamma)+(\gamma-1)}{(1+\beta)(1+\gamma)-(\gamma-1)}
$$




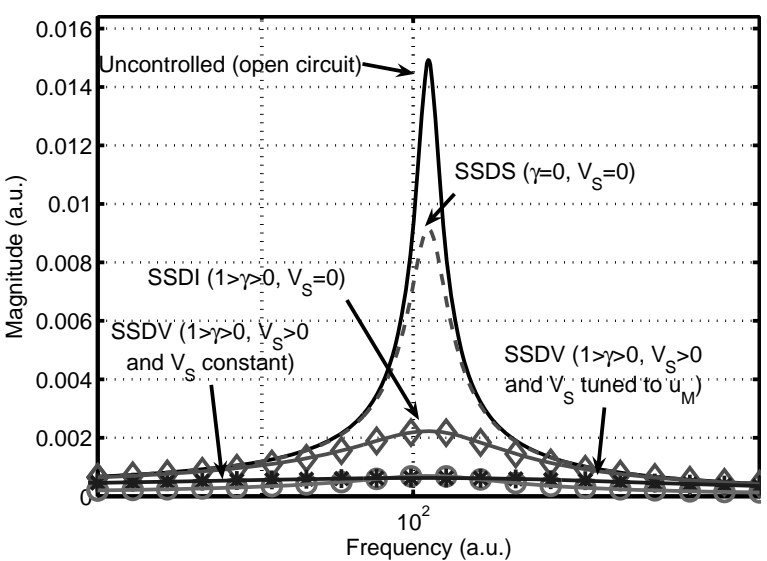

(a)

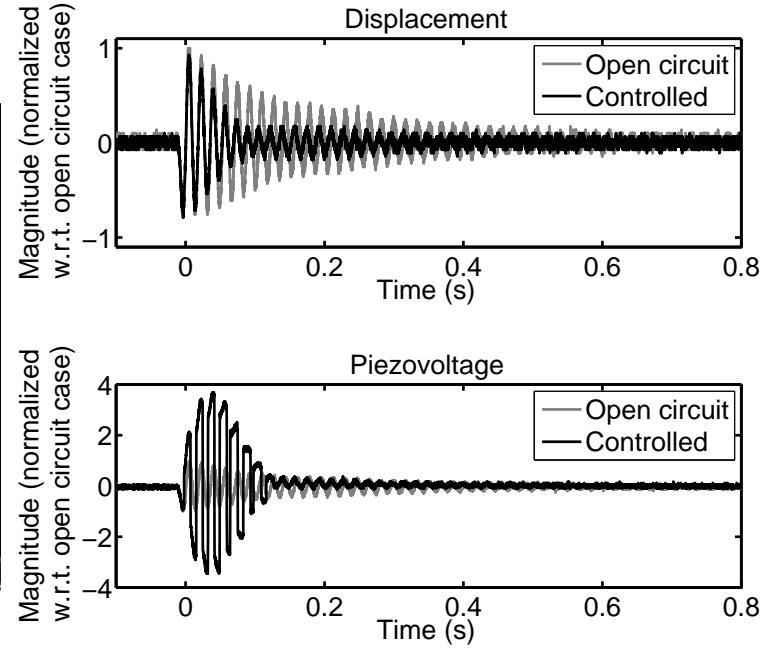

(b)

Figure 4. (a) Theoretical transfer functions of SSD techniques $\left(k^{2} Q_{M}=0.5, \gamma=0.8, V_{S}=60 \mathrm{~V}, \beta=3\right)(\mathrm{b})$ Experimental response to a pulse excitation using semi-passive $\operatorname{SSD}$ technique $\left(k^{2} Q_{M}=0.15, \gamma=0.78, V_{S}=0\right)$.

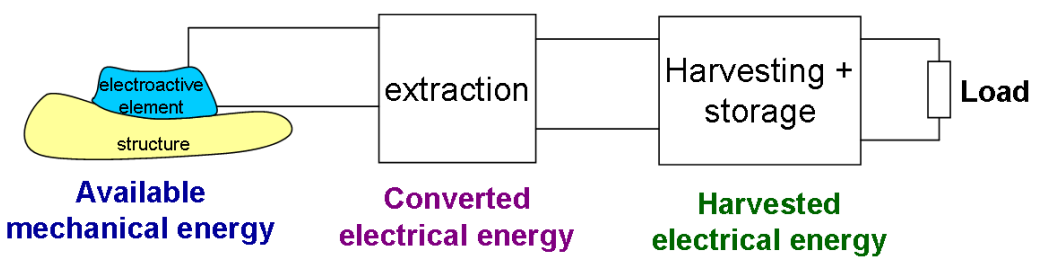

Figure 5. Energy harvesting chain.

A theoretical comparison of SSD performances is shown in Fig. 4(a).

Finally, for multimodal structures subjected to broadband excitation, it has been shown ${ }^{19}$ that selecting the switching extrema using a threshold leads to better performances rather than switching on all extrema.

In the case of pulse excitation, the energy conversion enhancement also allows a quick reduction of vibration due to the better conversion of mechanical energy into electrical energy (therefore less mechanical energy is available within the structure), as shown in Fig. 4(b).

\section{APPLICATION TO ENERGY HARVESTING: THE SSHI TECHNIQUE}

While the original energy conversion enhancement has initially been developed for vibration damping purposes, it is to store the converted energy for further electronic purpose. This is the aim of energy harvesting application. In addition of the enhancement of the conversion, another major topic when dealing with energy harvesting is to enhance the efficiency of the energy harvesting stage (Fig. 5).

The previously exposed conversion enhancement mechanism can be implemented in two ways for energy harvesting purposes: either the switching circuit is in parallel with the energy harvesting stage (parallel SSHI Fig. 6(a)), or in series (series SSHI - Fig. 6(b)).

In the parallel SSHI approach, the harvesting process intervenes when the absolute piezoelectric voltage is equal to the load voltage, until the current is non zero. After this process, the voltage is quickly reversed using the switching circuit (this operation does not occur in the standard approach). These two events (harvesting and voltage inversion) are merged in the case of series SSHI.

It is proposed here to derive the expected power output when the structure is subjected to a constant displacement magnitude $u_{M}$ at a frequency $f_{0}$. 


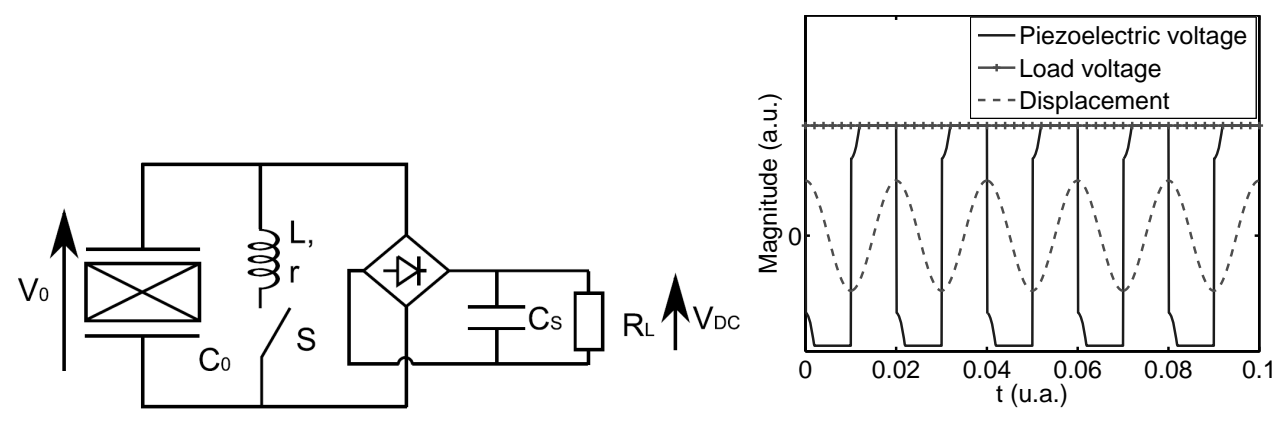

(a)
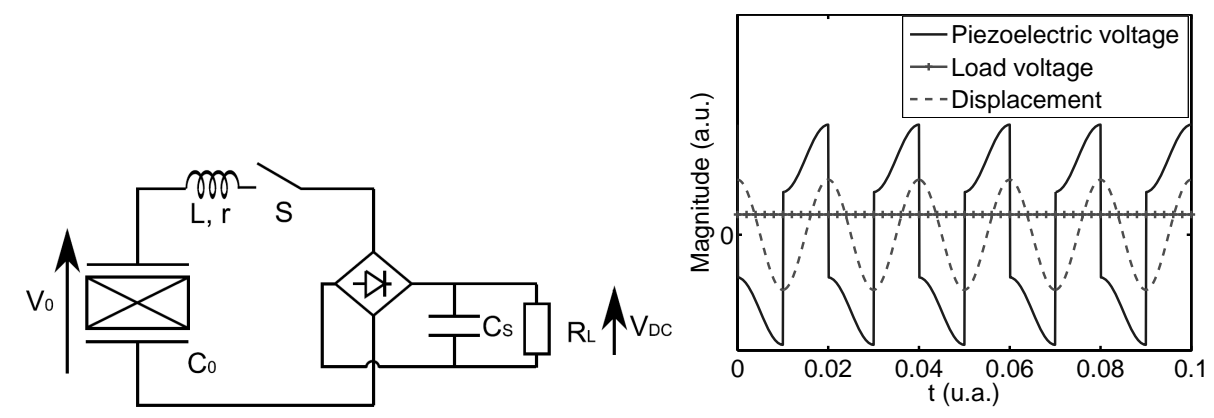

(b)

Figure 6. (a) Parallel SSHI schematics and associated waveforms (b) Parallel SSHI schematics and associated waveforms.

\subsection{Standard approach}

Using the standard interface, the harvesting process starts when the absolute piezovoltage is equal to the load voltage and ends when the displacement is equal to $\pm u_{M}$ (cancellation of the current). Considering a harvesting on positive piezovoltage, this corresponds to a voltage $-V_{D C}$ and a displacement of $-u_{M}$ at the end of the previous energy harvesting process, and a voltage $+V_{D C}$ at the beginning of the next harvesting event, corresponding to displacement value $u_{\text {stand }}$ is given by:

$$
u_{\text {stand }}=2 \frac{C_{0}}{\alpha} V_{D C}-u_{M}
$$

from Eq. (7). During an harvesting process and considering that the load voltage is constant, the energy amount is given by:

$$
E_{\text {stand }}=\int_{u_{\text {stand }}}^{u_{M}} \alpha V_{D C} d u=2 V_{D C}\left(\alpha u_{M}-C_{0} V_{D C}\right)
$$

As two energy harvesting events occur each vibrational period, the total output power is given by $P_{\text {stand }}=$ $2 f_{0} E_{\text {stand }}$. This power can also be expressed using $P_{\text {stand }}=V_{D C}{ }^{2} / R_{L}$. Therefore the power expression as a function of the load $R_{L}$ is given by:

$$
P_{\text {stand }}=\frac{\left(4 \alpha f_{0}\right)^{2} R_{L}}{\left(1+4 R_{L} C_{0} f_{0}\right)^{2}} u_{M}^{2}
$$




\subsection{Parallel SSHI}

In the case of the parallel SSHI, ${ }^{13,14}$ the energy harvesting process is almost the same, except that the voltage after the previous harvesting event (e.g. on negative voltage) is given by $\gamma V_{D C}$. Therefore the displacement $u_{\text {para }}$ at the beginning of a harvesting process on positive voltage is given by:

$$
u_{\text {para }}=(1-\gamma) \frac{C_{0}}{\alpha} V_{D C}-u_{M}
$$

yielding the harvested energy:

$$
E_{\text {para }}=\int_{u_{\text {para }}}^{u_{M}} \alpha V_{D C} d u=V_{D C}\left(2 \alpha u_{M}-(1-\gamma) C_{0} V_{D C}\right)
$$

And thus the output power as a function of the load $R_{L}$ is:

$$
P_{\text {para }}=\frac{\left(4 \alpha f_{0}\right)^{2} R_{L}}{\left[1+2(1-\gamma) R_{L} C_{0} f_{0}\right]^{2}} u_{M}^{2}
$$

\subsection{Series SSHI}

The harvesting principles of the series SSHI ${ }^{14,20,21}$ differs from the two previously exposed techniques. In this case, the energy is harvested at the same time the voltage is reversed, giving the harvested energy per switch event from Eq. (7):

$$
E_{\text {series }}=V_{D C} \int_{V_{M}}^{-V_{m}}-C_{0} d V
$$

where $V_{M}$ and $V_{m}$ denotes the absolute voltages before and after the switch event, respectively. These two values can be obtained from the expression of the inversion and when the piezoelement is left in open circuit:

$$
\left\{\begin{array}{l}
\gamma\left(V_{M}-V_{D C}\right)=V_{m}+V_{D C} \\
V_{M}-V_{m}=2 \frac{\alpha}{C_{0}} u_{M}
\end{array}\right.
$$

Combining Eqs. (22), (23) and using $P_{s e r}=V_{D C}{ }^{2} / R_{L}$, the expression of the output power as a function of the load $R_{L}$ yields:

$$
P_{\text {ser }}=\frac{\left[4(1+\gamma) \alpha f_{0}\right]^{2} R_{L}}{\left[(1-\gamma)+4(1+\gamma) R_{L} C_{0} f_{0}\right]^{2}} u_{M}^{2}
$$

\subsection{Comparison}

Fig. 7 depicts a comparison between the expected harvested powers in the three different cases. This figure shows that applying the nonlinear processing leads to an important energy gain, with a typical factor of 9 compared to the standard approach, using realistic components. The energy gain of the series SSHI is slightly lower than the one of the parallel SSHI. However, for large value of $\gamma($ i.e., $\gamma \rightarrow 1)$, the two output power are very similar. As well, the optimal load giving the maximal harvested power is changed according to the technique used. Particularly, the series SSHI presents a lower optimal load while the parallel SSHI optimal load is greater. These two values are distributed around the optimal load in the standard approach in a logarithmic fashion. It can also be noted that a deeper energy transfer analysis can be found in Ref. 17 .

The energy extraction process reduces the available mechanical energy as well. Hence, in a mechanical point of view, scavenging energy is equivalent to increase the losses in the mechanical structure, reducing the vibration magnitude. Therefore, if the system is submitted to a constant driving force, less mechanical energy is available, 


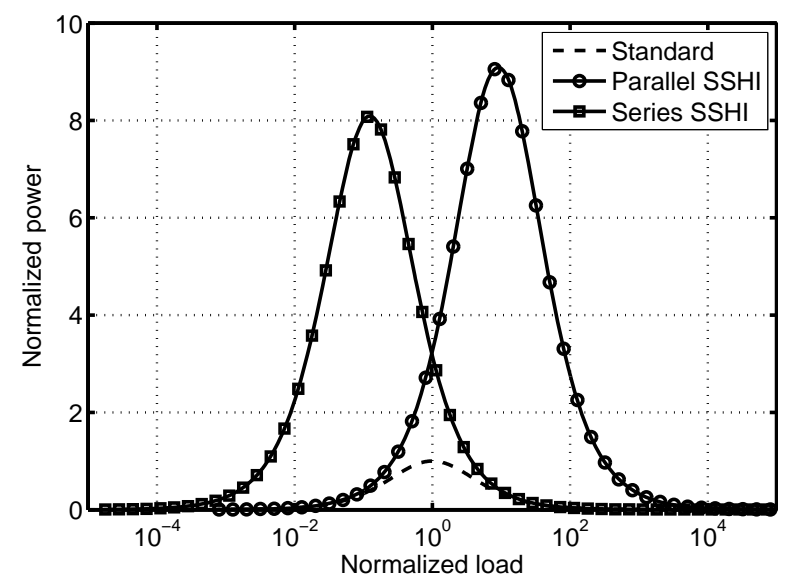

Figure 7. Comparison between energy harvesting techniques $(\gamma=0.78)$.

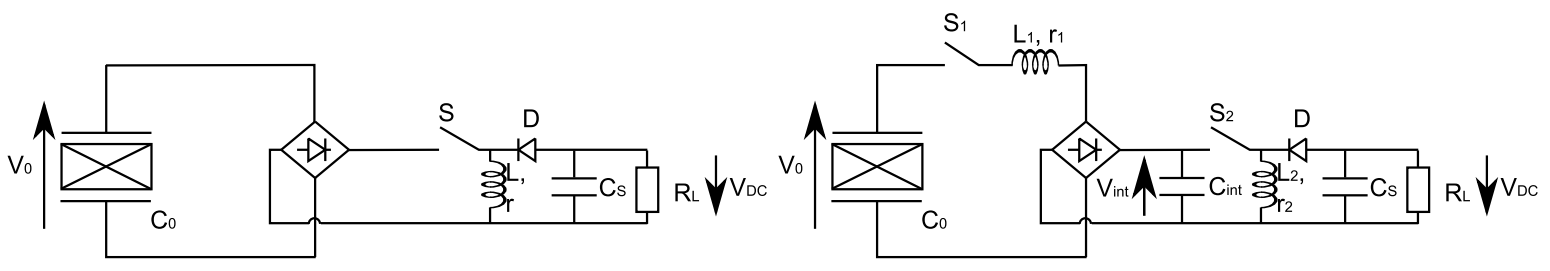

(a)

(b)

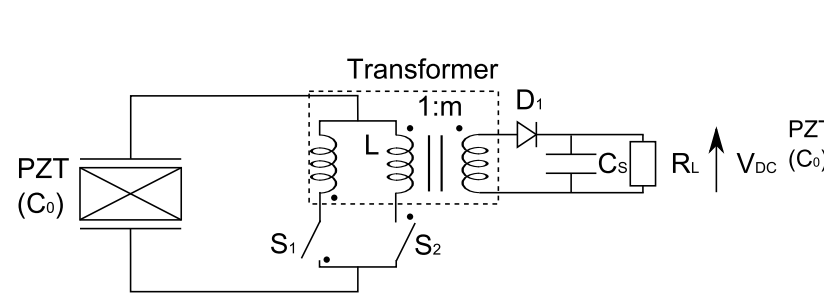

(c)

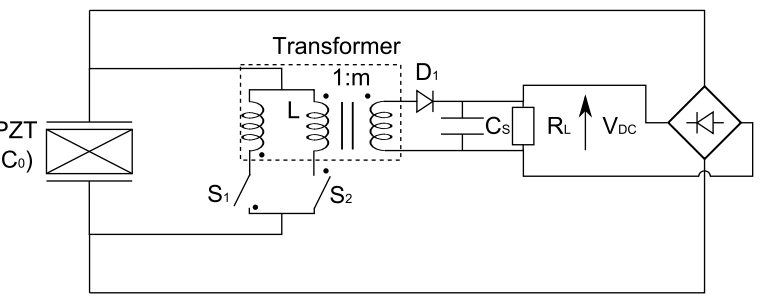

(d)

Figure 8. Nonlinear energy harvesting enhancement schematics (a) SECE (b) DSSH (c) SSHI-MR (d) Hybrid SSHI.

and therefore the output power is decreased. An analysis of the effect of harvesting on the vibration can be found in Ref. 14.

Finally, in a similar way than vibration damping, using nonlinear energy conversion enhancement leads to a great increase of the total amount of harvested energy in case of impulse excitation, as pointed out in Ref. 22, where it is shown that the approach allows scavenging up to 15 times more energy than the standard implementation when the system is submitted to a pulsed driving force.

\section{NONLINEAR ENERGY HARVESTING ENHANCEMENT}

\subsection{Principles}

The previous Section introduces the principles of energy harvesting using a nonlinear approach, resulting in a great improvement in terms of PEG output power. In this Section enhancements for nonlinear energy harvesting technique are proposed (Fig. 8). These are twofold:

- load independency (SECE, DSSH, Hybrid SSHI)

- low piezoelectric output voltage optimization (SSHI-MR) 
The SECE $^{14}$ (Fig. 8(a)) consists in extracting all the electrostatic energy available on the piezoelectric element each time the voltage reaches either a maximum or a minimum value. This electrostatic energy is converted in magnetic energy using the inductor, and then transferred to the storage capacitor back to the electrostatic form.

The principles of the $\mathrm{DSSH}^{23}$ (Fig. 8(b)) consists in combining the advantages of the SSHI (great gain) and SECE (load independency) by cite the extraction and harvesting stages. This is done using an intermediate capacitor $C_{\text {int }}$ which can be tuned to optimize the extracted power, and allows controlling the trade-off between voltage increase and energy extraction.

The SSHI-MR ${ }^{24}$ technique (Fig. 8(b)) has particularly been developed from the series SSHI architecture for systems with piezovoltage output. Indeed, in this case, the rectifier bridge is no longer negligible due to diode voltage gaps, not only reducing the effective amount of energy that can be harvested, but also limiting the inversion quality and therefore the gain of nonlinear techniques. The purpose of the SSHI-MR consists in adding a transformer between the piezoelement and the rectifying stage, thus reducing the voltage gaps seen by the piezoelectric element by the transformer ratio $m$.

Finally, the hybrid SSHI technique consists in taking into advantages the decoupling offered by the use of a transformer by combining the parallel SSHI and the SSHI-MR architectures, resulting in four energy extraction events a period instead of two. It can be noted that when the rectified voltage is important (i.e. important load values), the piezovoltage does not reaches $V_{D C}$, and only the SSHI-MR interface is operating.

Using similar approaches than in the previous Section, the associated power outputs as a function of the load $R_{L}$ for each of these architectures are given by:

$$
\begin{aligned}
& P_{S E C E}=4 f_{0} \gamma \frac{\alpha^{2}}{C_{0}} u_{M}^{2} \\
& P_{D S S H}=f_{0} \gamma_{C} \frac{1}{2} \frac{(1+\gamma)^{2}}{1-\gamma} \frac{\alpha^{2}}{C_{0}} u_{M}{ }^{2} \\
& P_{M R}=\frac{16 \frac{R_{L}}{m^{2}} f_{0}^{2}(1+\gamma)^{2}}{\left((1-\gamma)+4 f_{0} C_{0} \frac{R_{L}}{m^{2}}(1+\gamma)\right)^{2}} u_{M}^{2} \\
& \begin{cases}P_{\text {hybrid }}=\frac{\left(4 m^{2} f_{0} \alpha\right)^{2} R_{L}}{\left(m^{2}+2 R_{L} C_{0} f_{0}\left((1-\gamma) m^{2}+(1+\gamma)\right)\right)^{2}} u_{M}^{2} & \text { if } R_{L} \leq \frac{m^{2}}{2 C_{0} f_{0}(1+\gamma)(m-1)} \\
P_{\text {hybrid }} \frac{\left(4 m f_{0} \alpha(1+\gamma)\right)^{2} R_{L}}{\left((1-\gamma) m^{2}+4 R_{L} C_{0} f_{0}(1+\gamma)\right)^{2}} u_{M}{ }^{2} & \text { if } R_{L} \geq \frac{m^{2}}{2 C_{0} f_{0}(1+\gamma)(m-1)}\end{cases}
\end{aligned}
$$

\subsection{Comparison}

Fig. 9 depicts the different power outputs as a function of the load connected to the PEG. As before, the power outputs and loads have been normalized with regards to the maximal power output and optimal load in the standard case, respectively. This Figure demonstrates the effectiveness of the SECE and DSSH techniques in terms of load independency, while the gain in terms of harvested power is less than the SSHI approach. The SSHI-MR is very similar than the series SSHI for high-level vibrations (i.e. the diode bridge effect is negligible), but as demonstrated in Ref. 24, this technique is particularly interesting for low voltage output systems. By the way, it is possible to tune the optimal load through the transformer ratio, which is $m^{2}$ time greater than the optimal load of the series SSHI. Hence, the tuning of this load allows an increase of the bandwidth in terms of load, when associating the SSHI-MR with the parallel SSHI, leading to the concept of hybrid SSHI, which offers almost the same gain than the SSHI while greatly increasing the load bandwidth. 

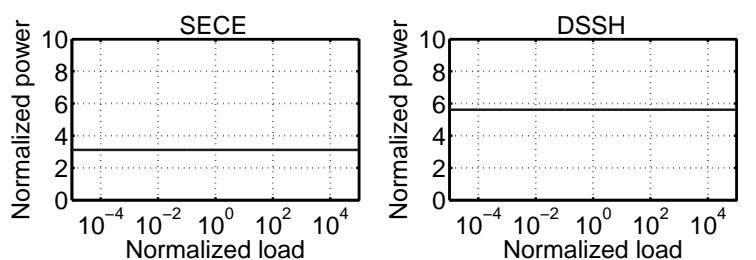

SSHI-MR
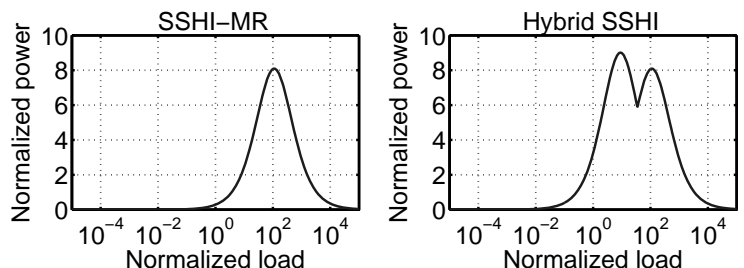

Figure 9. Comparison between enhanced energy harvesting techniques $(\gamma=0.78)$.

\section{CONCLUSION}

This paper presented a new approach for the physical interpretation of the energy conversion enhancement offered by nonlinear treatment. From this original approach, two applications have been recalled (vibration damping and energy harvesting), and new architectures aiming at either increasing the load bandwidth or counteracting the effect of discrete component voltage gaps have been exposed.

\section{REFERENCES}

[1] Guyomar, D., Jayet, Y., Petit, L., Lefeuvre, E., Monnier, T., Richard, C. and Lallart, M., "Synchronized Switch Harvesting applied to Self-Powered Smart Systems : Piezoactive Microgenerators for Autonomous Wireless Transmitters", Sensors and Actuators A : Physical 138(1), 151-160 (2007).

[2] Lallart, M., Guyomar, D., Jayet, Y., Petit, L., Lefeuvre, E., Monnier, T., Guy, P., and Richard, C., "Synchronized Switch Harvesting applied to Selfpowered Smart Systems : Piezoactive Microgenerators for Autonomous Wireless Receiver", Sensors and Actuators A : Physical 147(1), 263-272 (2008).

[3] Paradiso, J., A. and Feldmeier, M., "A Compact, Wireless, Self-Powered Pushbutton Controller", Proceedings of Ubicomp2001, 299-304 (2001).

[4] Krikke, J., "Sunrise for energy harvesting products", IEEE Pervasive Comput. 4, 4-35 (2005).

[5] Glynne-Jones, P., Beeby, S. P. and White, N., M., "Towards a piezoelectric vibration-powered microgenerator", IEE Proc. Sci. Meas. Technol. 148, 68-72 (2001).

[6] Ottman, G., K., Hofmann, H., F., Bhatt, A., C. and Lesieutre, G., A., "Adaptive piezoelectric energy harvesting circuit for wireless remote power supply", IEEE Trans. Power Electron. 17, 669-676 (2002).

[7] Anton, S., R. and Sodano, H., A., "A review of power harvesting using piezoelectric materials (2003-2006)", Smart Mater. Struct. 16, paper \#025009 (2007).

[8] Stephen, N., G., "On energy harvesting from ambient vibration", J. Sound Vib. 293, 409-425 (2005).

[9] Sodano, H., A., Inman, D., J. and Park, G., "A review of power harvesting from vibration using piezoelectric materials", Shock and Vib. Digest 36(3), 197-205 (2004).

[10] Priya, S., "Advances in energy harvesting using low profile piezoelectric transducers", J. Electroceram. 19, 165-182 (2007).

[11] Badel, A., Sebald, G., Guyomar, D., Lallart, M., Lefeuvre, E., Richard, C. and Qiu, J., "Wide band semiactive piezoelectric vibration control by synchronized switching on adaptive continuous voltage sources", J. Acoust. Soc. Am. 119(5), 2815-2825 (2006).

[12] Badel, A., Lagache, M., Guyomar, D., Lefeuvre, E. and Richard, C., "Finite element and simple lumped modeling for flexural nonlinear semi-passive damping", J. Intell. Mater. Syst. Struct. 18, 727-742 (2007).

[13] Guyomar, D., Badel, A., Lefeuvre, E. and Richard, C., "Towards energy harvesting using active materials and conversion improvement by nonlinear processing", IEEE Trans. Ultrason. Ferroelect. Freq. Contr. 52, 584-595 (2005). 
[14] Lefeuvre, E., Badel, A., Richard, C., Petit, L. and Guyomar, D., "A comparison between several vibrationpowered piezoelectric generators for standalone systems", Sens. Actuators A 126, 405-416 (2006).

[15] Lallart, M., Lefeuvre, E., Richard, C. and Guyomar, D., "Self-Powered Circuit for Broadband, Multimodal Piezoelectric Vibration Control", Sensors and Actuators A: Physical 143(2), 277-382 (2008).

[16] Lallart, M., Magnet, C., Richard, C., Lefeuvre, E., Petit, L., Guyomar, D. and Bouillault, F., "New Synchronized Switch Damping Methods Using Dual Transformations", Sensors and Actuators A : Physical 143(2), 302-314 (2008). doi : 10.1016/j.sna.2007.12.001

[17] Guyomar, D., Sebald, G., Pruvost, S., Lallart, M., Khodayari, A. and Richard, C., "Energy Harvesting From Ambient Vibrations and Heat", J. Intell. Mater. Syst., available online. doi : 10.1177/ 1045389X08096888

[18] Lallart, M., Badel, A. and Guyomar, D., "Non-Linear Semi-Active Damping Using Constant Or Adaptive Voltage Sources: A Stability Analysis", J. Intell. Mater. Syst. 19(10), 1131-1142 (2008).

[19] Guyomar, D. and Badel, A., "Nonlinear semi-passive multimodal vibration damping: An efficient probabilistic approach", J. of Sound and Vibration 294, 249-268 (2006).

[20] Taylor, G., W., Burns, J., R., Kammann, S., M., Powers, W., B. and Welsh, T., R., "The energy harvesting eel: A small subsurface ocean/river power generator", IEEE J. Oceanic Eng. 26, 539-547 (2001).

[21] Lallart, M. and Guyomar, D., "An optimized self-powered switching circuit for non-linear energy harvesting with low voltage output", Smart Mater. Struct. 17, paper \#035030, (2008).

[22] Badel, A., Lefeuvre, E., Richard, C. and Guyomar, D., "Efficiency enhancement of a piezoelectric energy harvesting device in pulsed operation by synchronous charge inversion", J. Intell. Mater. Syst. 16, 889-901 (2005).

[23] Lallart, M., Garbuio, L., Petit, L., Richard, C. and Guyomar, D., "Double Synchronized Switch Harvesting (DSSH): A New Energy Harvesting Scheme for Efficient Energy Extraction", IEEE Trans. Ultrason. Ferroelect. Freq. Contr. 55(10), 2019-2130 (2008).

[24] Garbuio, L., Lallart, M., Guyomar, D., Richard, C. and Audigier, D., "Mechanical energy harvester with ultra low threshold rectification based on SSHI non-linear technique", IEEE Trans. Indus. Electron., to be published. 